\title{
ESTIMATING THE OPERATOR'S PERFORMANCE TIME OF EMERGENCY PROCEDURAL TASKS BASED ON A TASK COMPLEXITY MEASURE
}

\author{
WONDEA JUNG* and JINKYUN PARK \\ Integrated Safety Assessment Dept. Korea Atomic Energy Research Institute \\ Daedeok-daero 989-111, Yuseong-gu, Daejeon, 305-353, Korea \\ "Corresponding author. E-mail : wdjung@kaeri.re.kr
}

Invited September14, 2011

Received November 29, 2011

Accepted for Publication February 27, 2012

It is important to understand the amount of time required to execute an emergency procedural task in a high-stress situation for managing human performance under emergencies in a nuclear power plant. However, the time to execute an emergency procedural task is highly dependent upon expert judgment due to the lack of actual data. This paper proposes an analytical method to estimate the operator's performance time (OPT) of a procedural task, which is based on a measure of the task complexity (TACOM). The proposed method for estimating an OPT is an equation that uses the TACOM as a variable, and the OPT of a procedural task can be calculated if its relevant TACOM score is available. The validity of the proposed equation is demonstrated by comparing the estimated OPTs with the observed OPTs for emergency procedural tasks in a steam generator tube rupture scenario.

KEYWORDS : Performance Time, Task Execution Time, Task Complexity, Procedural Task

\section{INTRODUCTION}

Reliable emergency response by human operators is a prerequisite to secure the safety of a nuclear power plant (NPP). There are some events that require prompt operator response to achieve optimal recovery and to maintain the plant within its design basis. Therefore, it is critical to understand the amount of time required to perform an emergency response when designing a NPP and emergency operating procedures (EOPs) as well as education and training programs[1][2].

For example, it is one of the assumptions used in the design of a NPP that operators should isolate a faulty steam generator (SG) within 30 minutes from initiation of the event, a steam generator tube rupture (SGTR). To validate the safety of the design, designer uses the operator's performance time (OPT) for isolating a faulty SG as an input to, or a requirement for, the safety analyses. Human reliability analysis (HRA) is another area that requires an OPT as either a direct or an indirect input to estimate human error probability [3].

Despite its significance, there is only a limited set of OPT data related to emergency procedural tasks in the NPPs. Due to the lack of data related to actual events that required an emergency response, a simulator may be used as an alternative to obtain OPT data. Several studies have been performed to collect data from a simulator to secure a technical basis for an HRA or to obtain data related to human performance [4][5]. Recently, a simulated experiment was performed by the Halden Reactor Project, which is an international research program that provides data and technical bases for an HRA [6][7]. A human performance database called OPERA (Operator Performance and Reliability Analysis) was developed by the authors to generate a portion of the input data for an HRA and to support research on human performance in NPPs [8][9].

However, there are difficulties with the broad use of that simulator data for applications in a systematic manner because they are able to support only a partial set of OPT, just for a limited amount of emergency procedural tasks of a few typical accident scenarios.

This paper introduces a method for estimating the OPT of a procedural task based on a measure of the task complexity, TACOM (Task Complexity) [10][11]. The method for estimating an OPT is an equation that is expressed by using the TACOM as a variable, and the OPT of a procedural task can be calculated if we know its TACOM score. 


\section{OPERA DATABASE}

The OPT is defined as a time duration between two points, from when a crew starts a procedural step to when it finishes it. The OPT of an emergency procedural task is required for the purpose of several applications, from safety analysis to operational management. However, a critical problem is that the amount of available OPT data is insufficient due to the infrequency of actual events or accidents. Therefore, a simulator was the only available and effective way to solve the problem of data shortage.

A human performance database called OPERA was developed by the authors [9]. The purpose of the OPERA development was to supply reliable information that could be used as a technical basis for examining human performance related problems including the HRA. Regarding the emergency tasks, full-scope training simulators were used to collect human performance data, including the OPT of the operators. More than 100 simulator records were secured from the training sessions of licensed MCR operators of reference plants for the OPERA. From the records, a set of information that serves a critical role in studying human performance was extracted by using two types of well-known analyses: protocol and timeline analysis [8].

Table 1 shows a summary of the OPT data for emergency tasks that are required for an SGTR scenario for reference plant A, which was generated from the OPERA database.

As shown in Table 1, the 'deliver a sufficient safety injection (SI) flow' task consists of two procedural steps: Steps 4 and 5. Thus, the OPT of the task, which is the time from the start of Step 4 to the end of Step 5, can be extracted from the OPERA DB.

Although the OPT data were used to supply plantspecific inputs for an HRA, however, the usage of the OPT was restrictive because the OPERA has only supplied the OPT data for a limited portion of the human failure events modeled in the probabilistic safety assessment (PSA) of the reference plant. Even if simulator data exists, the amount of data is statistically insufficient for some tasks.

For example, the E-3 (SGTR) procedure for the reference plant consists of 42 steps. However, the OPT data from OPERA are only available up to Step 14, as shown in Table 1. Due to the lack of OPT data in the OPERA, expert judgments were used for estimating OPT in order to support the HRA of the reference plants. However, a large amount of uncertainty is unavoidable when estimating human error probability, if many expert judgments are used to generate inputs for HRA, such as an OPT.

This paper proposes a method for estimating the OPT of a procedural task, such as the emergency response for the NPPs. The authors suggest an equation to calculate the OPT that is based on a measure of task complexity, TACOM [8][9]. The proposed method for estimating the OPT is an equation expressed with the TACOM, therefore, the OPT of a task can be calculated if we know its TACOM score.

\section{A method for OPT}

\subsection{Task Complexity Measure: TACOM}

The TACOM is a measure designed to quantify the complexity of a task that is prescribed in the procedures to guide the operations or emergency response of complex

Table 1. OPTs of the Emergency Procedural Tasks in SGTR Scenario.

\begin{tabular}{|c|c|c|c|c|c|}
\hline \multirow{2}{*}{ Procedure $^{\mathrm{a}}$} & \multirow{2}{*}{ Steps } & \multirow{2}{*}{ Task description } & \multirow{2}{*}{ Number of data sets ${ }^{\mathrm{b}}$} & \multicolumn{2}{|c|}{$\mathrm{OPT}^{\mathrm{c}}(\mathrm{sec})$} \\
\hline & & & & Average & Std. Dev. \\
\hline SPTA & $1 \sim 8^{\mathrm{d}}$ & Standard post trip action (SPTA) & 55 & 196.2 & 72.8 \\
\hline DA & $1 \sim 16$ & Diagnostic action (event diagnosis) & 15 & 195.9 & 106.7 \\
\hline \multirow{5}{*}{ E-3 } & $1 \sim 3$ & Reconfirm the entry conditions of E-3 & 3 & 1.2 & 4.6 \\
\hline & $4 \sim 5$ & Deliver a sufficient SI flow & 7 & 44.1 & 19.7 \\
\hline & $6 \sim 7$ & Check criteria for RCP stoppage & 9 & 89.0 & 66.2 \\
\hline & $8 \sim 10$ & $\begin{array}{l}\text { Initial cooling down the hot-leg } \\
\text { temperature of RCS }\end{array}$ & 4 & 169.0 & 66.7 \\
\hline & $11 \sim 14$ & Identify and isolate a faulty SG & 3 & 507.0 & 239.4 \\
\hline
\end{tabular}

aProcedure represents the relevant procedure. E-3 represents the procedure for an SGTR.

${ }^{b}$ Number represents the number of available data sets that were extracted from the simulator records.

'OPT represents the amount of time from the beginning of the first step to the end of the last step for a relevant task.

'Step \# written as ' $1 \sim 8$ ' means Step 1 through Step 8 of the SPTA procedure. 
systems [10]. It consists of five sub-measures that are related to a complexity factor that addresses a particular aspect of task complexity: (1) amount of information processed by the crew, (2) logical complications due to the logical relationships of the required actions, (3) amount of actions accomplished by the crew, (4) amount of system knowledge to recognize the problem space, and (5) amount of cognitive resources to establish an appropriate decision criterion.

Among the five factors, the first three factors represent the complexities that originate from the physical characteristics of the procedural step. The two remaining factors represent the complexities of a subjective load that originates from the cognitive characteristics of a task [11].

Based on the graph entropy concepts, TACOM was defined as a weighted Euclidean norm of five factors, as follows:

$T A C O M=\sqrt{(\alpha \times S I C)^{2}+(\beta \times S L C)^{2}+(\gamma \times S S C)^{2}+(\delta \times A H C)^{2}+(\varepsilon \times E D C)^{2}}$

- SIC: Step information complexity (SIC)

- SLC: Step logic complexity (SLC)

- SSC: Step size complexity (SSC)

- AHC: Abstraction hierarchy complexity (AHC)

- EDC: Engineering decision complexity (EDC)

- $\alpha, \beta, \gamma, \delta, \varepsilon$ are relative weights; even weights were used for this study.

The five sub-measures in Eq. (1) can be calculated by the first-order and the second-order graph entropies that were used to quantify the complexity of the software [12]. A high TACOM score implies a high cognitive complexity for the performance of a given task. Conducting a task with a higher TACOM score requires more time and results in a higher potential for error in emergency situations.

The validity of the TACOM has been confirmed by using a series of verification studies [10][11]. For one of the studies, the TACOM score was validated by comparing

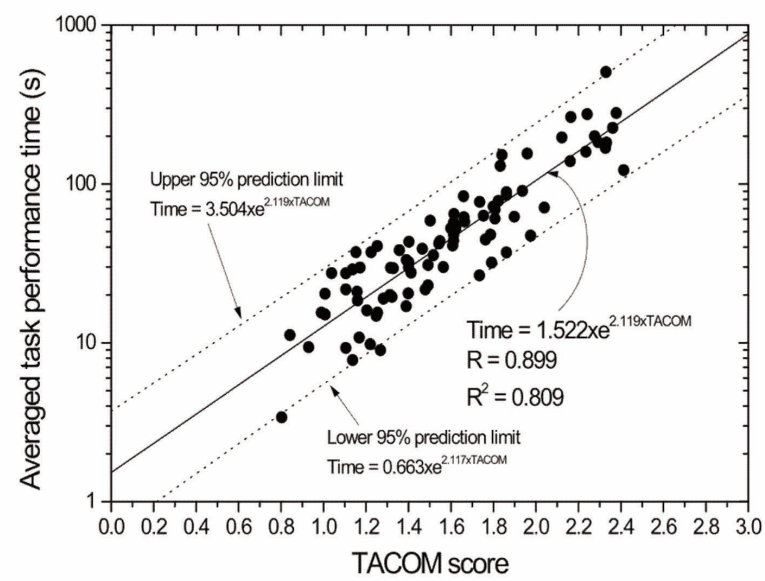

Fig. 1. Correlation between the OPTs and the Associated TACOM Scores. the OPTs for the emergency tasks that were obtained from simulator studies and the relevant TACOM scores. The authors calculated TACOM scores for a total of 86 emergency tasks for which the OPTs were available from the OPERA DB. A correlation between the OPT data and TACOM scores was investigated by using regression analysis. Fig. 1 shows the result of the regression analysis.

The validation studies showed that the TACOM score can be used to assess the level of complexity of a procedural task because there was a significant correlation between the averaged OPT data and the associated TACOM scores, as shown in Fig. 1

\subsection{An Equation for OPT}

As described in the previous section, the TACOM exhibits a high correlation with the OPT. Accordingly, the OPT of a procedural task can be produced by using the regression equation in Fig. 1, if the TACOM score of a task that is being considered is known. The equation for the OPT based on the TACOM is defined, as follows:

$$
\begin{aligned}
& \operatorname{OPT}_{\text {mean }}(i)=1.522 \times \operatorname{Exp}(2.119 \times \operatorname{TACOM}(i)) \\
& \operatorname{OPT}_{95 \%}(i)=3.504 \times \operatorname{Exp}(2.119 \times \operatorname{TACOM}(i)) \\
& \operatorname{OPT}_{5 \%}(i)=0.663 \times \operatorname{Exp}(2.119 \times \operatorname{TACOM}(i))
\end{aligned}
$$

where, OPTx ( $i$ ) is the estimated $x$ OPT of task $i$ ( $x$ is mean, 95\%, or 5\%) and TACOM(i) represents the TACOM score of task $i$.

Therefore, the OPT of a procedural task can be estimated by using Eq. (2) whenever its relevant TACOM score is available.

\subsection{A Validation Study}

To determine if the OPT equation is appropriate to estimate the execution time of a task, a validation study was performed by using another set of simulator data. In order to independently confirm the validity of the OPT equation, the crew's performance times that were gathered from reference plant B were compared to the associated OPT data that were derived from Eq. (2). An SGTR was then selected as the emergency scenario, and the simulator data were initially collected.

Table 2 shows a portion of the emergency tasks to be conducted by a crew in an SGTR scenario in reference plant B. OPT data for the nine emergency tasks were extracted with respect to the mean, standard deviation, and 95 upper percentile, with an assumed normal distribution. Table 2 also shows the associated TACOM scores and the relevant OPT data that were derived from Eq. (2). Finally, the estimated OPTs are compared to the 95 percentiles of the observed OPTs in Table 2, and Fig. 2 shows the result.

Fig. 2 shows that the 95 percentiles of the observed OPTs are distributed within the confidence intervals of 
Table 2. Observed and Estimated OPTs of SGTR Tasks in the Reference Plant B.

\begin{tabular}{|c|c|c|c|c|c|c|c|c|}
\hline \multirow[t]{2}{*}{ ID } & \multirow{2}{*}{$\begin{array}{l}\text { Procedure } \\
\text { and steps }\end{array}$} & \multirow{2}{*}{ Task description } & \multicolumn{2}{|c|}{$\begin{array}{l}\text { Observed OPT } \\
\quad(\mathrm{sec})\end{array}$} & \multirow{2}{*}{$\begin{array}{c}\text { TACOM } \\
\text { score }^{b}\end{array}$} & \multicolumn{3}{|c|}{ Estimated $\mathrm{OPT}^{\mathrm{c}}(\mathrm{sec})$} \\
\hline & & & Mean & $\mathrm{SD}^{\mathrm{d}}$ & & $5 \%$ & Mean & $95 \%$ \\
\hline 1 & $\mathrm{E}-0^{\mathrm{e}}, 1 \sim 4$ & Confirming immediate responses after reactor trip & 41.9 & 25.5 & 1.78 & 29.1 & 66.7 & 153.6 \\
\hline 2 & E-0, 5 6 & Confirming the isolation of essential valves & 12.0 & 2.9 & 1.60 & 19.8 & 45.6 & 104.9 \\
\hline 3 & E-0, 7 10 & Confirming the operation of essential pumps & 17.9 & 5.6 & 1.61 & 20.0 & 45.8 & 105.5 \\
\hline 4 & E-0, 11 14 & Verifying containment status & 33.9 & 22.3 & 1.79 & 29.4 & 67.6 & 155.5 \\
\hline 5 & E-0, 15 19 & Verifying the delivery of SI and AFW flow & 55.4 & 27.8 & 1.99 & 45.3 & 104.1 & 239.7 \\
\hline 6 & E-0, 19 21 & Verifying the status of RCS heat removal & 38.9 & 16.0 & 1.79 & 29.6 & 68.0 & 156.5 \\
\hline 7 & E-0, 22 23 & Entering E-3 procedure according to the status of SGs & 34.7 & 10.3 & 1.63 & 21.0 & 48.1 & 110.8 \\
\hline 8 & E- $3^{\mathrm{f}}, 1 \sim 4$ & Identifying and isolating faulty SGs & 97.0 & 28.6 & 2.47 & 123.8 & 284.2 & 654.3 \\
\hline 9 & $\mathrm{E}-3,5 \sim 13$ & Preparing the cool down of faulty SGs & 77.1 & 24.1 & 2.25 & 78.0 & 179.1 & 412.3 \\
\hline
\end{tabular}

${ }^{\mathrm{a} O b s e r v e d ~ O P T}$ is generated from simulator records of the reference plant $\mathrm{B}$.

${ }^{\mathrm{b}} \mathrm{TACOM}$ scores are quantified by equal weights.

${ }^{c}$ Estimated OPT is calculated by Eq.(2)

${ }^{\mathrm{d}}$ Standard deviation $(\mathrm{sec})$.

${ }^{\mathrm{e}} \mathrm{E}-0$ : procedure for an SPTA, ${ }^{\mathrm{f}} \mathrm{E}-3$ : procedure for an SGTR.

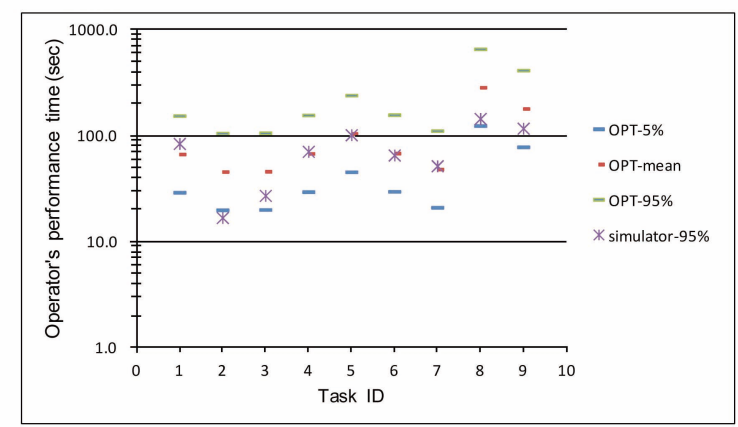

Fig. 2. Comparison between the Estimated OPTs and the 95 Percentiles of the Observed OPTs.

the estimated OPTs. Almost all of the 95 percentiles are less than or almost equal to the mean values of the estimated OPTs. Particularly, eight out of the nine tasks are located within the confidence intervals, and four of them are almost equal to the mean of the estimated OPTs. The estimated OPTs were compared to the 95 percentile of the observed OPTs because the OPTs should be determined as conservatively as possible, due to the fact that they would be used as input or a criterion for a safety analysis for the design or operation of the NPPs. Additionally, the performance time observed in the simulators tends to be slightly optimistic if it is compared to the actual performance time of a stressful accident scenario. Therefore, the estimated mean OPTs that were derived from Eq. (2) are conservative enough to be applied to the given applications, including the safety analysis of NPPs. Consequently, the OPT equation would be applicable to estimate the OPT of a procedural emergency task, especially for the Westinghouse 3 loops type plant which has almost the same type EOPs as the reference plant $\mathrm{A}$ and $\mathrm{B}$.

\section{OPT OF THE PROCEDURAL TASKS IN SGTR}

As an application of the method proposed in this paper, the OPTs for all of the emergency procedural tasks stipulated in the E-3 procedure of the reference plant were estimated by using Eq. (2). First, a task analysis was conducted for the E-3 procedure to identify and classify the procedural tasks, and the relevant TACOM scores for the pre-defined tasks were then calculated by using Eq. (1). Next, the OPTs for the pre-defined procedural tasks were estimated by using Eq. (2) with the relevant TACOM scores. Table 3 presents the result of the application.

The OPTs for all of the procedural tasks required for the SGTR scenario were estimated, as shown in Table 3, with respect to the means (5\%, and 95\%). One exception was the DA task, which was designed as a flow chart type of procedure. Because the TACOM measure is unable to be applied to a task that is implemented in the form of flow chart, the OPT of the DA task could not be derived from Eq. (2).

For the case of the SPTA task, the mean values of the observed and estimated OPTs were 196.2 and 190.64 seconds, respectively, and were very similar to each 
Table 3. OPTs of the Emergency Tasks in SGTR (E-3) Procedure.

\begin{tabular}{|c|c|c|c|c|c|c|}
\hline \multirow{2}{*}{$\begin{array}{l}\text { Procedure } \\
\text { and steps }\end{array}$} & \multirow[t]{2}{*}{ Task description } & \multicolumn{2}{|c|}{$\begin{array}{l}\text { Observed OPT based on } \\
\text { simulator data }(\mathrm{sec})\end{array}$} & \multicolumn{3}{|c|}{$\begin{array}{l}\text { Estimated OPT based on } \\
\text { the TACOM score }(\mathrm{sec})\end{array}$} \\
\hline & & Mean & SD & $5 \%$ & Mean & $95 \%$ \\
\hline SPTA $^{\text {a }}, 1-9$ & Standard post trip action (SPTA) & 196.2 & 72.8 & 78.25 & 190.64 & 439.32 \\
\hline $\mathrm{DA}^{\mathrm{b}}, 1-16$ & Diagnostic action (event diagnosis) & 195.9 & 106.7 & - & - & - \\
\hline $\mathrm{E}-3,1-3$ & Reconfirm the entry conditions of E- 3 & 1.2 & 4.6 & 0.63 & 1.52 & 3.5 \\
\hline E-3, 4-5 & Deliver a sufficient SI flow & 44.1 & 19.7 & 18.95 & 46.12 & 106.22 \\
\hline $\mathrm{E}-3,6-7$ & Check criteria for RCP stoppage & 89.0 & 66.2 & 32.16 & 78.32 & 180.41 \\
\hline $\mathrm{E}-3,8-10$ & Initial cooling down the hot-leg temperature of RCS & 169.0 & 66.7 & 86.99 & 211.93 & 488.42 \\
\hline E-3, 11-14 & Identify and isolate a faulty SG & 507.0 & 239.4 & 186.24 & 351.93 & 294.67 \\
\hline E-3, 15-18 & Terminate HPSI/LPSI if necessary & & & 45.13 & 109.91 & 253.23 \\
\hline E-3, 19 & Check P/T of RCS within criteria & & & 27.73 & 67.53 & 155.54 \\
\hline E-3, 20-21 & Check levels of SG and CST & & & 14.39 & 35.02 & 80.64 \\
\hline E-3, 22 & Monitor radiation level of secondary side & & & 6.04 & 14.70 & 33.83 \\
\hline E-3, 23 & Inject borated water into RCS to secure subcooled margin & & & 6.86 & 16.69 & 38.41 \\
\hline E-3, 24 & Maintain PZR level (15 70\%) & & & 24.42 & 59.47 & 136.97 \\
\hline $\mathrm{E}-3,25$ & Initiate RCS cooling for SDC & & & 14.70 & 35.77 & 82.37 \\
\hline $\mathrm{E}-3,26-27$ & Check boron density and opening RCP breaker & & & 12.94 & 31.5 & 72.54 \\
\hline E-3, 28 & Maintain level of faulted SG & & & 30.83 & 75.07 & 172.93 \\
\hline E-3, 29-30 & Restart CVCS letdown and checking PZR level & & & 22.44 & 54.64 & 125.84 \\
\hline E-3, 31 & Recover of offsite power if necessary & & & 6.44 & 15.66 & 36.05 \\
\hline E-3, 32-34 & Check RCS condition and restarting RCP & & & $79 / 93$ & 194.72 & 448.73 \\
\hline E-3, 35 & Sampling for radiation monitoring of secondary side & & & 0.63 & 1.52 & 3.50 \\
\hline E-3, 36-37 & Remove air bubble of RCS & & & 122.06 & 297.42 & 685.55 \\
\hline E-3, 38 & Start cool down and depressurization of faulted SG & & & 22.44 & 54.64 & 125.84 \\
\hline E-3, 39 & Isolate SIT & & & 10.93 & 26.59 & 61.22 \\
\hline E-3, 40 & Starting lineup of LTOP valves & & & 7.79 & 18.95 & 43.62 \\
\hline E-3, 41 & Terminating ESFAS if necessary & & & 15.99 & 38.93 & 89.66 \\
\hline E-3, 42 & Starting SDC operation & & & 23.91 & 58.22 & 134.10 \\
\hline
\end{tabular}

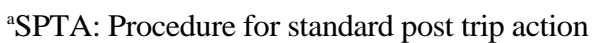

${ }^{\mathrm{b}} \mathrm{DA}$ : Procedure for diagnostic action

other. The observed and estimated OPTs for the tasks that are stipulated in the early phase of an SGTR event were also similar to each other. For the case of the 'deliver a sufficient SI flow' and 'check criteria for RCP stoppage' tasks in the E-3 procedure, a large difference was not observed between the two OPTs (the observed and the estimated values).
However, a considerable gap was observed between the two OPTs for the 'identify and isolated a faulty SG' task. This implies that the task required a more comprehensive cognitive and decision-making process for assessing the entire plant status, identifying a faulty SG, and planning on how to isolate it. The task has a unique cognitive characteristic that is not fully considered in the TACOM 
measure, which resulted in an underestimation of the OPT. From a performance time perspective, the 'terminate HPSI/LPSI if necessary', 'check RCS condition and restarting RCP', and 'remove air bubble of RCS' tasks require a longer performance time compared to other tasks in the SGTR scenario.

In some cases, an OPT needs to be used carefully because it does not include the time required to reach a predefined condition for a task. The time to start or finish a task can increase in some of the emergency tasks if the operators must wait for a specific plant condition. Tasks engaged in the early phase of an emergency scenario should typically be implemented promptly to mitigate the occurring event. Therefore, the task completion time can be estimated by using only OPT data. For example, for the case of an SGTR, the time to complete an 'isolation of a faulty SG' task can be calculated by summing the average values of the OPTs from the SPTA to Step 14 of the E-3 in Table 1 because those tasks are implemented promptly without any waiting time. On the other hand, tasks that are stipulated in the later portion of an emergency procedure, which are typically required if a plant reaches a stable condition, should typically be started after the predefined condition is attained. For example, if a crew opens a valve after the pressure of a tank reaches a certain level, then the task completion time will be longer than its estimated OPT, if a period of time is required to reach a set point. Accordingly, to derive the time to start or finish a task due to a reactor trip, both the OPT and the time to reach a predefined condition for the task should be considered.

\section{CONCLUSION}

For the design of the EOPs or safety analyses of a NPP, it is critical to understand the amount of time required to perform an emergency response task in a high-stress situation. However, due to the lack of observed actual data, the OPT of a task is highly dependent upon expert judgment.

This paper suggested an equation with TACOM to analytically estimate the OPT of a procedural task. This equation was derived based on the results of previous studies that demonstrated a high positive correlation between the OPT observed from a simulator and the relevant TACOM score of an emergency task. Accordingly, the OPT was directly calculated by using a regression equation between the OPT and TACOM score. This implies that the OPT of a procedural task can be estimated if the relevant TACOM score is available.

As an application, Eq. (2) was applied to estimate the OPTs for all of the emergency tasks stipulated in the E-3 (SGTR) procedure of the reference plant. The E-3 procedure consists of 42 steps; however, the OPTs from OPERA are only available for 14 steps of the procedure. As shown in
Table 3, the estimated OPTs for all of the steps in the E-3 procedure were generated by using the equation proposed in the paper.

The validation study showed that the 95 percentiles of the observed OPTs were distributed within the confidence intervals of the estimated OPTs. Almost all of the 95 percentiles were less than or almost equal to the mean values of the estimated OPTs, which is reasonable if the usage of the OPTs in the field of safety analysis is considered. The estimated OPT can be used as a reference value for a crew's OPT of a procedural task for an SGTR scenario in the NPPs. Accordingly, Eq. (2) could be applicable for estimating the OPT of a procedural emergency task in the NPPs.

\section{ACKNOWLEDGEMENT}

This research was supported by a Nuclear Research \& Development Program of the National Research Foundation (NRF) grant funded by the Korean government. (Grant code: 2012-011506).

\section{REFERENCES}

[ 1 ] USNRC, "Human Factors Engineering Program Review Model," NUREG/CR-0711, USNRC (2004).

[2 ] NEA, "Research Strategies for Human Performance," NEA /CSNI/R(97)24, Nuclear Energy Agency (1998).

[3 ] B. Kirwan, A guide to practical human reliability assessment, Taylor \& Francis (1994).

[4 ] G. W. Hannaman, A. J. Spurgin, and Y. D. Lukic, "Human cognitive reliability model for PRA analysis," Drafts report NUS-4531, EPRI Project RP2170-3 (1984).

[ 5 ] Westinghouse Owner's Group, "Emergency operator response time assessment program," WCAP-14996, Westinghouse Energy System (1997).

[ 6 ] Halden Reactor Project, "Halden reactor Project Program: Proposal for the three year period 2006-2008 man, technology, organization (MTO) program," HP-1165, IFE (2006).

[7] B. Andreas, K. Laumann, P. Braarud, and S. Massaiu, "Methodology for improving HRA by simulator studies," Proc. Int. Conf. PSAM8, New Orleans, USA, 2006.

[ 8 ] W. Jung, J. Park, J. Kim, and J. Ha, “Analysis of an Operators' Performance Time and Its Application to a Human Reliability Analysis in Nuclear Power Plants," IEEE Trans. Nucl. Sci., Vol. 54, pp. 1801-1811 (2007).

[9] J. Park and W. Jung, "OPERA - A Human Performance Database under Simulated Emergencies of Nuclear Power Plants," Reliab. Eng. Syst. Saf., Vol. 92, pp. 503-519 (2007).

[10] J. Park and W. Jung, "A Study on the Development a Task Complexity Measure for Emergency Operating Procedure of Nuclear Power Plants," Reliab. Eng. Syst. Saf., Vol. 92, pp. 1102-1116 (2007).

[11] J. Park and W. Jung, "A Study on the Validity of a Task Complexity Measure for Emergency Operating Procedure of Nuclear Power Plants - Comparing Task Complexity Scores with Two Sets of Operator Response Time Data Obtained under a Simulated SGTR," Reliab. Eng. Syst. Saf., Vol. 93, pp. 557-566 (2008).

[12] K. Lew, "Software Complexity and Its Impact on Software Reliability," IEEE Trans. Software Eng., Vol. 14, pp. 16451655 (1988). 